\title{
Treating allergic diseases in pregnancy
}

\author{
Birgit Pfaller ${ }^{1}$, Sarah Bendien ${ }^{2}$, Agnès Ditisheim³ ${ }^{3}$ and Thomas Eiwegger ${ }^{4}$ \\ ${ }^{1}$ Karl Landsteiner University of Health Science, University Hospital St. Pölten-Lilienfeld \\ ${ }^{2}$ Haga Teaching Hospital \\ ${ }^{3}$ Hôpital de la Tour \\ ${ }^{4}$ The Hospital for Sick Children
}

February 25, 2021

\begin{abstract}
Allergic diseases like asthma, allergic rhinitis, or food allergy have a high prevalence in women of childbearing age and may affect up to $30 \%$ of this age group. A multitude of immunological changes characterizes pregnancy to create the optimal milieu for the unborn child. Both these immunological changes and pre-conceptional, sub-optimal disease control may affect the severity of the respective allergic disease manifestations during pregnancy and pose a risk for mother and child. Due to apparent limitations in conducting clinical trials, safety data on anti-allergic drugs during pregnancy are limited. This lack of clinical evidence demands to counsel between potential and known risks and benefits of anti-allergic drugs. This includes the potential of disease aggravation in the absence of treatment. By doing so, informed decisions and shared decision-making is facilitated. In particular, in patients with severe asthma, education about the risk of uncontrolled asthma for mother and child should be part of regular care. This review focuses on the management of allergic diseases during pregnancy, maternal counseling, and available information/evidence regarding allergic diseases' management and treatment during pregnancy. Furthermore, we discuss the challenges of treating patients with allergic diseases and covid-19 during pregnancy.
\end{abstract}

\section{Hosted file}

ReviewAllergicDiseasePreg2_2021.pdf available at https://authorea.com/users/397976/articles/ 510696-treating-allergic-diseases-in-pregnancy 